
\title{
Multimorbidité et nouvelle structure du programme
}

Le problème des maladies chroniques et de la multimorbidité sera au cœur de la prochaine assemblée annuelle de 2013. Nous prévoyons d'y aborder ce sujet sous l'angle médical et d'en évaluer les conséquences au niveau de la planification des soins. La structure du programme va également changer pour apporter une nette amélioration à la vue d'ensemble et répondre aux besoins variés des participants.

\section{Edouard Battegay}

Prof. Dr, membre du comité de la Société Suisse de Médecine Interne Générale (SSMI)
Correspondance: SSM

Case postale 422 CH-4008 Bâle Tél. 0614678551 Fax 0614678556 info[at]sgim.ch
La multimorbidité, ou présence simultanée de plusieurs troubles et maladies chroniques, est le tableau clinique le plus fréquemment rencontré de nos jours. En raison de l'évolution démographique et des progrès continus de la médecine, il faut encore compter avec une augmentation du nombre de patients multimorbides. Dans des situations souvent complexes, les généralistes praticiens et hospitaliers doivent poser un diagnostic différentiel, faire un triage et effectuer un traitement tout en travaillant avec un arrièreplan de multimorbidité. En parallèle, les guidelines usuels se focalisent sur le traitement de pathologies isolées et ne prennent en considération les comorbidités que de façon marginale. Peu de recherches se sont consacrées jusqu'ici aux modifications de traitement à entreprendre en présence de deux maladies ou davantage. Dans les cas de maladies chroniques, il ne reste souvent aux généralistes hospitaliers et aux médecins de famille qu'à recourir à des décisions individuelles basées sur leur expérience et leur intuition. Il ne faut cependant pas oublier de tenir compte des maladies chroniques et des multimorbidités au sein de la future planification des soins médicaux. Nous aborderons en profondeur ces questionnements à l'occasion de divers exposés.

\section{Objectif de l'assemblée annuelle}

L'objectif de l'assemblée annuelle de 2013 sera par conséquent de traiter des thèmes des maladies chroniques et de la multimorbidité qui font partie de notre quotidien. Cet objectif répond-il aux attentes des futurs participants à l'assemblée annuelle de 2013 de la SSMI? L'évaluation des réponses aux questionnaires distribués aux internistes lors de l'assemblée générale de 2012 reflète une réalité professionnelle multiple, voire hétérogène. Certains désirent une remise à jour rapide des thèmes de l'an passé, d'autres voudraient approfondir des questionnements scientifiques. D'autres collègues voudraient qu'on leur offre des débats et des présentations aussi réalistes que possible sur la manière d'agir face à certains problèmes importants dans la pratique.

Nous avons donc décidé de subdiviser le programme principal en quatre itinéraires didactiques formatés en fonction des différents besoins. Pour permettre une vue d'ensemble sur la complexité des manifestations parallèles, chaque itinéraire disposera de son propre auditoire. Les participants pourront en outre aisément bifurquer d'un itinéraire vers l'autre, car l'horaire des pauses est homogène. Nous espérons ainsi répondre aux attentes de nombreux collègues, et nous les invitons à visiter les autres auditoires pour se laisser surprendre et explorer de nouveaux sujets médicaux et non médicaux. L'assemblée annuelle a été conçue comme une manifestation de rencontre et de réunion. C'est la condition sine qua non pour qu'elle reste la «réunion de classe des internistes». Les itinéraires du programme seront:

Tendances - un choix de sujets traitant de la politique de la santé ou de problèmes généraux d'ordre sociopolitique. L'objectif sera ici d'approfondir la réflexion sur la direction prise par la médecine au sein de son environnement social.

Mises à jour - cet itinéraire présentera des sujets de médecine interne générale et des thèmes spécialisés, dans le but de donner une vue d'ensemble de l'évolution dans les différentes disciplines. Il est prévu d'y intégrer des contributions des sociétés de disciplines invitées.

Science et recherche - ces séminaires continueront à interroger des thèmes actuels de la médecine; chaque sujet sera traité de façon approfondie et sous différents angles par plusieurs intervenants. Il sera fait référence à la recherche actuelle.

Méthodes - il s'agit de donner aux médecins des recommandations fondées sur les données factuelles pour répondre aux défis diagnostiques ou thérapeutiques de la pratique quotidienne. Contrairement aux ateliers, cette partie du programme se déroulera sous forme d'exposé structuré. Elle réservera néanmoins un temps suffisant aux questions des participants. Dans cette manifestation, nous visons une collaboration étroite avec la SSMG.

Informations actuelles sur www.congrex.ch/fr/ sgim2013 ou www.sgim.ch/fr/manifestations-de-formation-continue/ssmi-congres-annuel/2013/ 